\title{
Coping with Loss Aversion in the Newsvendor Model
}

\author{
Jianwu Sun and Xinsheng Xu \\ Department of Mathematics, Binzhou University, Binzhou 256603, China \\ Correspondence should be addressed to Jianwu Sun; sjwbz1964@163.com
}

Received 31 October 2014; Accepted 12 April 2015

Academic Editor: Juan R. Torregrosa

Copyright (C) 2015 J. Sun and X. Xu. This is an open access article distributed under the Creative Commons Attribution License, which permits unrestricted use, distribution, and reproduction in any medium, provided the original work is properly cited.

\begin{abstract}
We introduce loss aversion into the decision framework of the newsvendor model. By introducing the loss aversion coefficient $\lambda$, we propose a novel utility function for the loss-averse newsvendor. First, we obtain the optimal order quantity to maximize the expected utility for the loss-averse newsvendor who is risk-neutral. It is found that this optimal order quantity is smaller than the expected profit maximization order quantity in the classical newsvendor model, which may help to explain the decision bias in the classical newsvendor model. Then, to reduce the risk which originates from the fluctuation in the market demand, we achieve the optimal order quantity to maximize CVaR about utility for the loss-averse newsvendor who is risk-averse. We find that this optimal order quantity is smaller than the optimal order quantity to maximize the expected utility above and is decreasing in the confidence level $\alpha$. Further, it is proved that the expected utility under this optimal order quantity is decreasing in the confidence level $\alpha$, which verifies that low risk implies low return. Finally, a numerical example is given to illustrate the obtained results and some management insights are suggested for the loss-averse newsvendor model.
\end{abstract}

\section{Introduction}

In recent years, the study about the newsvendor model has attracted the attentions of many researchers and it has been applied to many fields, such as production plan and yield management [1-3]. In the classical newsvendor model, the newsvendor needs to choose an order quantity before the selling season to maximize his/her expected profit under a stochastic market demand. Here, if the newsvendor's order quantity is bigger than the realized market demand, the newsvendor has to dispose the excess order as a loss; otherwise if the newsvendor's order quantity is smaller than the realized market demand, the newsvendor will be punished for the lost sales. As we all know, the expected profit maximization order quantity has been well documented in the newsvendor literature. However, some recent studies found that the realized order quantity of the manager in practice always deviates from the expected profit maximization order quantity above, which is referred as "decision bias" in the newsvendor problem (see [4]). For example, Brown and Tang [5] conducted a simple experiment by giving a single-period inventory problem to $250 \mathrm{MBA}$ students and 6 professional buyers who order fashion items and observed that both groups select their order quantities less than their expected profit maximization order quantities. Then, to explain such a phenomenon, some researchers paid more attention to study the "decision bias" in the newsvendor model and propose many alternative methods to study the newsvendor model. For example, some researchers introduced other risk references of the newsvendor such as risk-aversion to study the optimal order quantity decision of the newsvendor model and obtained some useful results (see [6-11]). Moreover, some other researchers introduced other objectives rather than expected profit maximization for the newsvendor to choose an optimal order quantity. For example, many people proposed the objective of maximizing the probability of achieving a certain profit level for the newsvendor model, which is extensively adopted by the managers in real life (see $[12,13])$. Besides, some studies asserted the existence of a "pull to center" bias in the newsvendor problem; that is, the retailers in practice often select a quantity between the expected profit-maximizing order quantity and the mean of the market demand [14-16]. Above all, to help the newsvendors with different decision criterions and/or preferences to select the order quantities more accurately, it is not surprising to see that many extensions of the classical newsvendor model have 
appeared; see Khouja [17] and Qin et al. [18] for a detailed literature review.

In the real world, it is known that some decision makers are more averse to the losses coming from the decisions than being attracted to the same sized profits from the decisions, which is referred to as "loss aversion" in the decision theory. In fact, loss aversion is both intuitively attractive and well supported in various fields (e.g., finance and economics). For example, the empirical studies by Shapira [19] based on interviews with 50 American and Israeli executives and MacCrimmon and Wehrung [20] based on questionnaires from 509 high-level executives of American and Canadian firms both show that managers' decision-making behaviors in the real world are always consistent with loss aversion. Moreover, a stream of literature closely related to the successful applications of loss aversion can be found in various areas, such as financial markets [21], marketing [22], organizational behavior [23], labor supply [24], and supply chain management [25-28]. In fact, it is pointed out that the loss aversion mentioned above also exists in the newsvendor model. For example, since the loss comes from the lost sales that ranges from profit loss on the scale to some unspecific loss of goodwill of the customers and always has an more important influence on the benefit of the newsvendor, especially the long-term benefits of the newsvendor, then some newsvendors are more averse to the loss coming from the excess order or lost sales when the selling season is due. But so far, compared with the deep study and wide applications of loss aversion in other fields, it has been little applied to the decision framework of the newsvendor model. Then, such a study about the loss aversion in the newsvendor model is necessary and meaningful, especially for the loss-averse newsvendors. Besides, the loss aversion of the newsvendor may be one of the multiple choices that can help explain the decision bias in the newsvendor model.

In light of the above successful applications of loss aversion, this paper then introduces loss aversion into the decision framework of the newsvendor model. First, we present the gain obtained in the selling season and the loss comes from the excess order when the selling season is due for the newsvendor separately and then introduce a novel utility function for the loss-averse newsvendor by introducing the loss aversion coefficient $\lambda$. Then, we obtain the optimal order quantity for the loss-averse newsvendor who is risk-neutral to maximize his/her expected utility first. It is found that this optimal order quantity is smaller than the expected profit maximization order quantity, and the more loss-averse the newsvendor is, the less products he/she orders. Further, to measure and control the risk originating from the fluctuation in market demand, by adopting the CVaR measure which is widely used in finance management, we achieve the optimal order quantity for the loss-averse newsvendor who is riskaverse to maximize his/her CVaR about utility, which can guarantee the maximum expectation of the utility which is below a certain quantile. It is checked that this optimal order quantity is smaller than the optimal order quantity for the loss-averse newsvendor to maximize the expected utility above, and the more risk-averse the newsvendor is, the less products he/she orders. Finally, a numerical example is given to show the obtained results and some management insights are suggested for the optimal order quantity decisions in the loss-averse newsvendor model. Our study thus contributes to the newsvendor literature in two main aspects. First, we prove that the optimal order quantity for the loss-averse newsvendor to maximize his/her expected utility is smaller than the expected profit maximization order quantity in the classical newsvendor model; this may help to explain the decision bias in the classical newsvendor model. Second, it is found that, in the loss-averse newsvendor model, for the risk-averse newsvendor, his/her optimal order quantity to maximize CVaR about utility is decreasing in the confidence level $\alpha$, and the expected utility under such an optimal order quantity is decreasing in the confidence level $\alpha$ as well. This implies that if the loss-averse newsvendor chooses an order quantity to reduce/control the potential risk, he/she will expect a lower utility, which verifies the following fact: high risk, high return; low risk, low return.

The rest of this paper is organized as follows. In the following section, we give a detailed description on the loss-averse newsvendor model and present some preliminaries about $\mathrm{VaR}$ and $\mathrm{CVaR}$. Section 3 studies the optimal order quantity decisions for the loss-averse newsvendor to maximize his/her expected utility and CVaR about utility, respectively; the properties of the two optimal order quantities are presented as well. Section 4 gives a numerical example and sensitivity analysis to verify the obtained results in Section 3, and some management insights for the loss-averse newsvendor model are suggested by the numerical results, with the conclusions given in Section 5.

\section{Preliminaries}

In this section, we will give a detailed description on the lossaverse newsvendor model studied in this paper and present some basic knowledge about the CVaR measure in financial risk management.

2.1. Presentation and Motivation. For the newsvendor model, suppose that the market demand $\xi$ is a random variable, and its probability density function and cumulative distribution function are $f(\cdot)$ and $F(\cdot)$, respectively. Without loss of generality, it is supposed that $F(0)=0, F(+\infty)=1, F(\cdot)$ is continuously differentiable and increasing, and thus the inverse of $F(\cdot)$ exists. First, we present the gain obtained in the selling season and the loss comes from the excess order when the selling season is due for the newsvendor. Here, for an order quantity $q$ of the newsvendor and the realized value $D$ of $\xi$, the gain of the newsvendor obtained in the selling season can be given as

$$
G(q)=(p-c) \min \{q, D\} .
$$

In the above equality, $p$ is the retail price of unit product, and $c$ is the wholesale price of unit product from the supplier. It is pointed out that the above gain $G(q)$ comes from the sales of the products and is realized in the selling season, while the following introduced loss for the newsvendor occurs when the selling season is due. Here, when the selling season is due, there may be some products that can not be sold. For such 
a case, the loss of the newsvendor from the excess order can be given as

$$
L(q)=(c-r)(q-D)^{+}
$$

where $X^{+}=\max \{X, 0\}$. Here, $r$ is the salvage price of unit product which can not be sold. Without loss of generality, it is assumed that $p \geq c \geq r \geq 0$ holds. Evidently, the newsvendor likes to take the gain $G(q)$, while he/she is averse to the loss $L(q)$. Then, for the realized market demand $D$, by (1) and (2), we introduce the following utility function for the loss-averse newsvendor to select an order quantity $q$ :

$$
\begin{aligned}
U(q) & =G(q)-\lambda L(q) \\
& =(p-c) \min \{q, D\}-\lambda\left[(c-r)(q-D)^{+}\right],
\end{aligned}
$$

where $\lambda \geq 1$ is the loss aversion coefficient. The loss aversion coefficient $\lambda$ indicates the newsvendor's aversion level to the loss $L(q)$, and the bigger the loss aversion coefficient $\lambda$ becomes, the more loss-averse the newsvendor is. This utility function $U(q)$ indicates that the newsvendor is loss-averse; that is, compared with the satisfaction from the gain obtained in the selling season, the newsvendor loses more satisfaction in suffering the same sized loss when the selling season is due.

In the following, we first obtain the optimal order quantity for the loss-averse newsvendor to maximize the expected utility. However, in recent years, some unpredictable disasters (e.g., earthquakes and economic crisis) disrupt the supply chain operations repeatedly, and this makes the retailers in reality become more sensitive to the market demand and more averse to the risk originating from the fluctuation in the market demand. Then, many researchers paid attention to the risk analysis and risk control in the newsvendor model by introducing various risk measures $[6,7,11]$. Recently, some people introduced the CVaR measure in financial management to cope with the risk aversion in the newsvendor problem and got some useful results $[8,9]$. These papers proved that the CVaR measure is efficient in coping with the risk coming from the fluctuation in the market demand for the newsvendor model. For example, in Chen et al. [8], it is concluded that the optimal order quantity for a risk-averse newsvendor to maximize his/her CVaR about profit is smaller than the risk-neutral newsvendor's expected profit maximization order quantity, which provides a possible choice that can help explain decision bias in the newsvendor model. Following this idea, to control and reduce the risk originating from the fluctuation in the market demand for the loss-averse newsvendors, we will incorporate the CVaR measure into the decision framework of the loss-averse newsvendor model.

2.2. Risk Measure: VaR and CVaR. Before Conditional Valueat-Risk (CVaR) measure was introduced, Value-at-Risk (VaR) measure is widely used in the risk management in finance. Here, for a decision $x$ and the random variable $\xi$, let $l(x)$ be the loss from the decision $x$ for the decision maker. Then, for a given confidence level $\alpha$, the Value-at-Risk (VaR) about $l(x)$ is given as

$$
\operatorname{VaR}_{\alpha}[l(x)]=\inf \{y \in R \mid \operatorname{Pr}\{l(x) \leq y\} \geq \alpha\},
$$

where $\operatorname{Pr}\{l(x) \leq y\}$ denotes the probability of $l(x)$ not exceeding the value $y$. Then, the value of $\operatorname{VaR}_{\alpha}[l(x)]$ represents the minimum loss from the decision $x$ for the decision maker under the confidence level $\alpha$. However, it is found that the VaR measure has some undesirable mathematical characteristics, such as nonsubadditivity and nonconvexity, which always hinder its efficient usage in the applications [29, 30]. Then, Rockafellar and Stanislav [31] and Rockafellar and Uryasev [32] introduced the Conditional Value-at-Risk (CVaR) measure into the financial risk management. CVaR is a downside risk measure which captures a risk of the loss going above to some target level. For a given confidence level $\alpha$, the CVaR about the loss $l(x)$ above is defined as

$$
\mathrm{CVaR}_{\alpha}[l(x)]=E\left[l(x) \mid l(x) \geq \operatorname{VaR}_{\alpha}[l(x)]\right],
$$

where $\operatorname{VaR}_{\alpha}[l(x)]$ is defined by (4). Here, the $\operatorname{CVaR}_{\alpha}[l(x)]$ represents the expected value of the loss which exceeds the quantile $\operatorname{VaR}_{\alpha}[l(x)]$. By minimizing the CVaR objective, the decision maker can obtain an optimal solution, which minimizes the expectation of the loss that exceeds the quantile $\operatorname{VaR}_{\alpha}[l(x)]$. The CVaR measure has some attractive properties such as coherence and convexity, which makes it widely used in financial risk management as compared to VaR measure. To compute, Rockafellar and Stanislav [31] introduced the following auxiliary function $F(x, u)$ :

$$
F(x, u)=u+\frac{1}{1-\alpha} E[l(x)-u]^{+},
$$

and they proved that the optimal solution to minimize the objective $\mathrm{CVaR}_{\alpha}[l(x)]$ can be obtained by minimizing the above function $F(x, u)$.

\section{Optimizing the Objectives about Utility $U(q)$}

In this section, we will introduce different objectives about the utility function $U(q)$ introduced in the above section for the loss-averse newsvendors with different risk preferences and give the optimal order quantity decisions for the newsvendors to optimize these objectives.

3.1. Maximizing the Expected Utility Function $E[U(q)]$. For the loss-averse newsvendor problem, since the realized market demand $D$ can not be observed before the selling season starts, then the newsvendor can not observe his/her realized utility $U(q)$ from the order quantity $q$. For such a case, the conventional approach to analyze the newsvendor model is based on assuming that the newsvendor is risk-neutral and makes the order quantity decision to maximize his/her expected performance. Following this idea, in this subsection, we will discuss the optimal order quantity decision to maximize the expected utility $E[U(q)]$ ( $E$ is the expectation operator) for the loss-averse newsvendor who is risk-neutral.

Theorem 1. For the loss-averse newsvendor model, the optimal order quantity for a risk-neutral newsvendor to maximize the expected utility $E[U(q)]$ is given by

$$
q^{*}=F^{-1}\left[\frac{p-c}{p-c+\lambda(c-r)}\right] \text {. }
$$


Proof. For a given order quantity $q$ of the newsvendor and the realized market demand $D$, it follows from (3) that

$$
U(q)=(p-c) \min \{q, D\}-\lambda\left[(c-r)(q-D)^{+}\right]
$$

Then, it follows from $\min \{q, D\}=q-(q-D)^{+}$that

$$
U(q)=(p-c) q-[p-c+\lambda(c-r)](q-D)^{+} .
$$

Then the expectation of $U(q)$ is given by

$$
\begin{aligned}
E[U(q)]= & (p-c) q \\
& -[p-c+\lambda(c-r)] \int_{0}^{q}(q-t) d F(t),
\end{aligned}
$$

which implies

$$
\frac{\partial E[U(q)]}{\partial q}=(p-c)-[p-c+\lambda(c-r)] F(q) .
$$

Then, it follows that

$$
\frac{\partial^{2} E[U(q)]}{\partial q^{2}}=-[p-c+\lambda(c-r)] f(q)<0,
$$

which implies that $E[U(q)]$ is concave in $q$. Then it follows from $\partial E[U(q)] / \partial q=0$ that $E[U(q)]$ attains the maximum in

$$
q^{*}=F^{-1}\left[\frac{p-c}{p-c+\lambda(c-r)}\right] .
$$

This completes the proof.

By Theorem 1, in the loss-averse newsvendor model, the optimal order quantity for a loss-averse newsvendor to maximize his/her expected utility $E[U(q)]$ is decided by the retail price $p$, the wholesale price $c$, the salvage price $r$, and the loss aversion coefficient $\lambda$. In particular, if it satisfies $\lambda=1$, which implies the loss-averse newsvendor turns to be lossneutral, then it follows from Theorem 1 that

$$
q^{*}=F^{-1}\left[\frac{p-c}{p-c+\lambda(c-r)}\right]=F^{-1}\left[\frac{p-c}{p-r}\right],
$$

which is same as the expected profit maximization order quantity. Moreover, since it satisfies

$$
\begin{aligned}
& \frac{p-c}{p-r}-\frac{p-c}{p-c+\lambda(c-r)} \\
& \quad=\frac{(\lambda-1)(p-c)(c-r)}{(p-r)(p-c+\lambda(c-r))} \geq 0,
\end{aligned}
$$

which implies

$$
\frac{p-c}{p-r} \geq \frac{p-c}{p-c+\lambda(c-r)}
$$

then it follows that

$$
F^{-1}\left[\frac{p-c}{p-r}\right] \geq F^{-1}\left[\frac{p-c}{p-c+\lambda(c-r)}\right] .
$$

That is to say, the optimal order quantity for a loss-averse newsvendor to maximize his/her expected utility $E[U(q)]$ is smaller than the optimal order quantity for a newsvendor to maximize his/her expected profit in the classical newsvendor model. The intuition is clear for this result: if the newsvendor is loss-averse and he/she is more averse to the loss coming from the excess order when the selling time is due than the newsvendor who aims to maximize the expected profit, then it is better for him/her to order less products to avoid or reduce the loss from excess order. Then, this result provides a possible reason for the existence of decision bias in the newsvendor model and may help to explain the experiment conducted by Brown and Tang [5], in which the experimental results show that the experiment participants select their order quantities less than their expected profit maximization order quantities.

By Theorem 1, the following results are obvious.

Corollary 2. For the loss-averse newsvendor model, the optimal order quantity $q^{*}$ for a loss-averse newsvendor to maximize his/her expected utility $E[U(q)]$ is increasing in the retail price $p$ and the salvage price $r$ and decreasing in the wholesale price $c$, respectively.

It is not surprising to see that this result holds, and this result also holds in the classical newsvendor model when the newsvendor selects an optimal order quantity to maximize his/her expected profit. Here, it is pointed out that, for any fixed $\lambda$, if it satisfies $r \rightarrow c$, we have $F^{-1}[(p-c) /(p-c+$ $\lambda(c-r))] \rightarrow F^{-1}(1)=+\infty$, which implies that if the excess order can be salvaged at a higher price, then the newsvendor will order more products. Particularly, if it satisfies $r=c$, it follows that $F^{-1}[(p-c) /(p-c+\lambda(c-r))]=F^{-1}(1)=+\infty$, which implies that if the excess order can be salvaged at the wholesale price, then there is no loss for the excess order, and the loss-averse newsvendor will order products as many as possible.

Corollary 3. For the loss-averse newsvendor model, the optimal order quantity $q^{*}$ for a loss-averse newsvendor to maximize his/her expected utility $E[U(q)]$ is decreasing in the loss aversion coefficient $\lambda$.

By this result, if the loss-averse newsvendor becomes more loss-averse to the loss from the excess order, he/she will order less products. Particularly, let $\lambda \rightarrow+\infty$, and then it follows that $q^{*}=F^{-1}[(p-c) /(p-c+\lambda(c-r))] \rightarrow F^{-1}(0)=0$. That is to say, if the newsvendor is loss-averse enough, then he will not order a product and no longer sell this product.

In this subsection, for the loss-averse newsvendor model, we obtain the optimal order quantity decision for a lossaverse newsvendor to maximize his/her expected utility $E[U(q)]$. However, it is pointed out that this expected utility maximization measure ignores the risk originating from the fluctuation in the market demand, which is not enough to some loss-averse newsvendors who are risk-averse. Moreover, if the variance of this expected utility is large, then the obtained expected utility maximization order quantity may lead to an unpredictably loss for the loss-averse newsvendor. 
In view of this critical issue, in the following subsection, we will incorporate the risk aversion into the decision framework of the loss-averse newsvendor model and introduce the Conditional Value-at-Risk criterion which is widely used in the financial risk management to measure and control the risk which originates from the fluctuation in the market demand for the risk-averse newsvendors.

3.2. Maximizing $C V a R$ about Utility $U(q)$. For the order quantity $q$ of the loss-averse newsvendor and the utility $U(q)$ from this order quantity, under the given confidence level $\alpha$, we first define the $\operatorname{VaR}$ about $U(q)$ for the loss-averse newsvendor as follows:

$$
\operatorname{VaR}_{\alpha}[U(q)]=\sup \{y \in R \mid \operatorname{Pr}\{U(q) \geq y\} \geq \alpha\}
$$

which represents the maximum utility that the loss-averse newsvendor can obtain under the confidence level $\alpha$. The $\mathrm{CVaR}$ measure is a downside risk measure which captures the risk of the profit (or utility) going down to the target level, while the profit (or utility) above this target level is ignored. This is acceptable since the profit (or utility) above the target level can not be regarded as a risk to be hedged, but more pleasant gain. Then, taking $\operatorname{VaR}_{\alpha}[U(q)]$ as the target level, the $\mathrm{CVaR}$ about the utility $U(q)$ for the loss-averse newsvendor is given as

$$
\mathrm{CVaR}_{\alpha}[U(q)]=E\left[U(q) \mid U(q) \leq \operatorname{VaR}_{\alpha}[U(q)]\right],
$$

which represents the expected value of the utility which is below the target level $\mathrm{VaR}_{\alpha}[U(q)]$. By maximizing this $\mathrm{CVaR}$ objective of $\mathrm{CVaR}_{\alpha}[U(q)]$, we can obtain an optimal order quantity for the loss-averse newsvendor to maximize the expected value of the utility which is below the quantile $\operatorname{VaR}_{\alpha}[U(q)]$ under the given confidence level $\alpha$. Then, we have the following result about the optimal order quantity for the risk-averse newsvendor to maximize this CVaR objective.

Theorem 4. For the loss-averse newsvendor model, the optimal order quantity for a loss-averse newsvendor to maximize his/her CVaR about $U(q)$ is given by

$$
q^{\alpha}=F^{-1}\left[\frac{(1-\alpha)(p-c)}{p-c+\lambda(c-r)}\right] \text {. }
$$

Proof. See the Appendix.

Here, it is easily checked that if it satisfies $\alpha=0$, which implies the loss-averse newsvendor who is risk-averse turns to be risk-neutral, then it follows from Theorem 4 that $q^{\alpha}=$ $q^{*}$ holds. Similar to the results in Corollaries 2 and 3, we have the following results about the optimal order quantity $q^{\alpha}$.

Corollary 5. For the loss-averse newsvendor model, the optimal order quantity $q^{\alpha}$ for a loss-averse newsvendor to maximize his/her CVaR about utility $U(q)$ is increasing in the retail price $p$ and the salvage price $r$ and decreasing in the wholesale price $c$, respectively.
In the loss-averse newsvendor model, by Corollary 2, if it satisfies $r=c$, we have $q^{*}=F^{-1}(1)=+\infty$ and the lossaverse newsvendor who aims to maximize his/her expected utility will order products as many as possible. However, it is important to point out that this property does not hold for the loss-averse newsvendor who is risk-averse. By Corollary 5, for the risk-averse newsvendor $(\alpha \neq 0)$, if it satisfies $r=c$, we have $q^{\alpha}=F^{-1}[(1-\alpha)(p-c) /(p-c)]=F^{-1}(1-\alpha) \neq F^{-1}(1)$. This result shows that even though the excess order can be salvaged at the wholesale price, the loss-averse newsvendor who is risk-averse still considers the fluctuation in the market demand and selects the order quantity prudently.

Corollary 6. For the loss-averse newsvendor model, the optimal order quantity $q^{\alpha}$ for a loss-averse newsvendor to maximize his/her CVaR about utility $U(q)$ is decreasing in the loss aversion coefficient $\lambda$.

This result shows that, to reduce the risk coming from the fluctuation in the market demand, the loss-averse newsvendor will order less products if he/she becomes more lossaverse.

Corollary 7. For the loss-averse newsvendor model, the optimal order quantity $q^{\alpha}$ for a loss-averse newsvendor to maximize his/her CVaR about utility $U(q)$ is decreasing in the confidence level $\alpha$.

The confidence level $\alpha$ reflects the degree of risk aversion of the loss-averse newsvendor, and the bigger the confidence level $\alpha$ becomes, the more risk-averse the lossaverse newsvendor is. This result shows that, in the lossaverse newsvendor model, if the loss-averse newsvendor becomes more risk-averse, then the optimal order quantity $q^{\alpha}$ for him/her to maximize his/her CVaR about utility $U(q)$ decreases. It can be explained as follows: since there is no shortage penalty for the lost sales, then the risk mainly comes from the excess order. Therefore, if the loss-averse newsvendor becomes more risk-averse, then it is better for him/her to order less products to avoid or reduce the loss from excess order.

As mentioned above, if the confidence level $\alpha$ becomes bigger, the loss-averse newsvendor becomes more risk-averse and will order less products. Then, how does the expected utility $E[U(q)]$ under the optimal order quantity $q^{\alpha}$ of a lossaverse newsvendor changes with the growth of the confidence level $\alpha$ ? We have the following result to address this issue.

Corollary 8. For the loss-averse newsvendor model, the expected utility $E\left[U\left(q^{\alpha}\right)\right]$ of the loss-averse newsvendor under the optimal order quantity $q^{\alpha}$ is decreasing in the confidence level $\alpha$.

Proof. By (10), we have

$$
\begin{aligned}
E[U(q)]= & (p-c) q \\
& -[p-c+\lambda(c-r)] \int_{0}^{q}(q-t) d F(t) .
\end{aligned}
$$


It follows that

$$
\begin{aligned}
& \frac{\partial E\left[U\left(q^{\alpha}\right)\right]}{\partial \alpha} \\
& \quad=\left[p-c-(p-c+\lambda(c-r)) F\left(q^{\alpha}\right)\right] \frac{\partial q^{\alpha}}{\partial \alpha} .
\end{aligned}
$$

Since it satisfies $q^{\alpha} \leq q^{*}$ and $q^{*}=F^{-1}[(p-c) /(p-c+\lambda(c-r))]$, it follows that

$$
\begin{aligned}
& p-c-(p-c+\lambda(c-r)) F\left(q^{\alpha}\right) \\
& \quad \geq p-c-(p-c+\lambda(c-r)) F\left(q^{*}\right)=0 .
\end{aligned}
$$

Then, it follows from (22), (23), and Corollary 7 that

$$
\frac{\partial E\left[U\left(q^{\alpha}\right)\right]}{\partial \alpha} \leq 0,
$$

which proves that $E\left[U\left(q^{\alpha}\right)\right]$ is decreasing in the confidence level $\alpha$. This completes the proof.

By this result, in the loss-averse newsvendor model, if the risk-averse newsvendor decreases his/her order quantity to reduce the risk coming from the fluctuation in the market demand, he/she will expect a lower utility. This verifies that high return follows high risk, while low risk means low return.

\section{Numerical Results}

In this section, we will give two examples to show the results obtained in Section 3 and present some management insights for the loss-averse newsvendor model.

Example 9. For the loss-averse newsvendor model, suppose the market demand $\xi$ subjects to the uniform distribution $U(0,1000)$. Moreover, the other parameters are given as $p=$ $10, c=6$, and $r=2$. For these parameters, let us compute the optimal order quantities $q^{*}$ and $q^{\alpha}$ obtained in Section 3 for the loss-averse newsvendor and give a sensitivity analysis.

First, let $\lambda=2$ and $\alpha=0.5$, we compute the optimal order quantities $q^{*}$ and $q^{\alpha}$ with different value of the retial price $p$, the wholesale price $c$, and the salvage price $r$ for the lossaverse newsvendor separately, and the results are given in Figures 1, 2, and 3, respectively.

By Figures 1, 2, and 3, it is easily checked that $q^{*}$ and $q^{\alpha}$ both are increasing in the retail price $p$ and the salvage price $r$ and decreasing in the wholesale price $c$. Moreover, it satisfies $q^{*}>q^{\alpha}$ for different value of $p, c$, and $r$.

Further, let $p=10, c=6, r=2$, and $\alpha=0.5$; we compute the optimal order quantities $q^{*}$ and $q^{\alpha}$ with different value of the loss aversion coefficient $\lambda$ for the loss-averse newsvendor, and the result is given in Figure 4. By Figure 4, the optimal order quantities $q^{*}$ and $q^{\alpha}$ both are decreasing in the loss aversion coefficient $\lambda$. Besides, it also satisfies $q^{*}>q^{\alpha}$ for different value of $\lambda$.

Then, let $p=10, c=6, r=2$, and $\lambda=2$; we compute the optimal order quantities $q^{*}$ and $q^{\alpha}$ with different value of

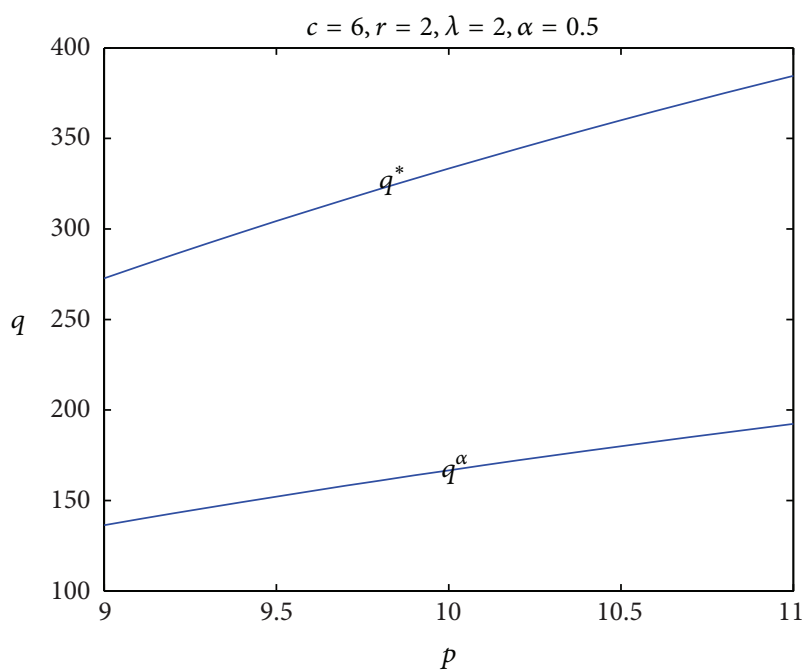

Figure 1: Optimal order quantities $q^{*}$ and $q^{\alpha}$ with different value of $p$.

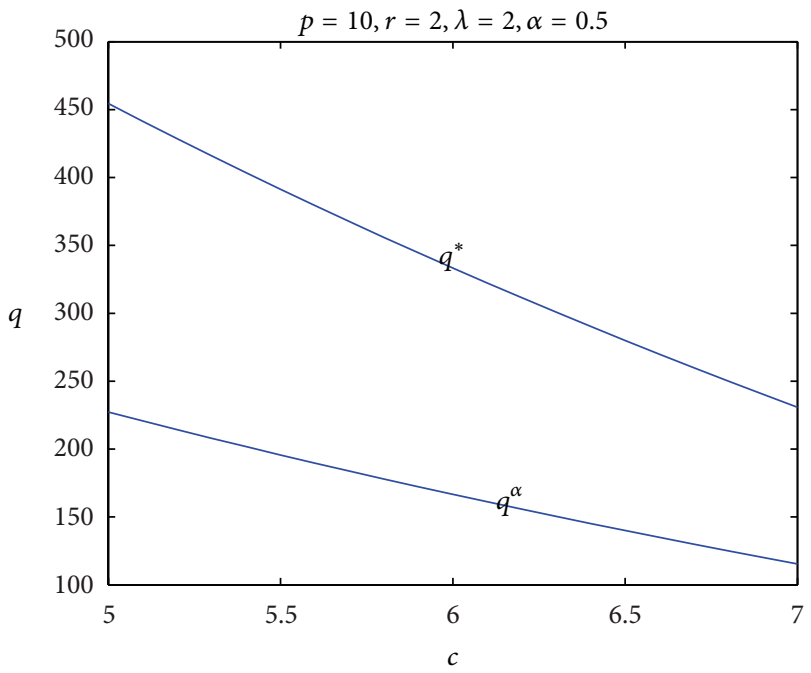

FIgURE 2: Optimal order quantities $q^{*}$ and $q^{\alpha}$ with different value of $c$.

the confidence level $\alpha$ for the loss-averse newsvendor, and the result is given in Figure 5. By Figure 5, the optimal order quantity $q^{*}(\alpha=0)$ stays the same and the optimal order quantity $q^{\alpha}$ is decreasing in the confidence level $\alpha$. Besides, it also satisfies $q^{*}>q^{\alpha}$ for different value of $\alpha$.

Finally, let $p=10, c=6, r=2$, and $\lambda=2$, we compute the expected utilities $E\left[U\left(q^{*}\right)\right]$ and $E\left[U\left(q^{\alpha}\right)\right]$ with different value of the confidence level $\alpha$ for the loss-averse newsvendor, and the result is given in Figure 6. By Figure 6, the expected utility $E\left[U\left(q^{*}\right)\right](\alpha=0)$ stays the same and the expected utility $E\left[U\left(q^{\alpha}\right)\right]$ is decreasing in the confidence level $\alpha$. Besides, it also satisfies $E\left[U\left(q^{*}\right)\right] \geq E\left[U\left(q^{\alpha}\right)\right]$ for different value of $\alpha$.

Example 10. For the loss-averse newsvendor model, suppose the market demand $\xi$ subjects to the normal distribution $N\left(1000,100^{2}\right)$. Moreover, the other parameters are given as 


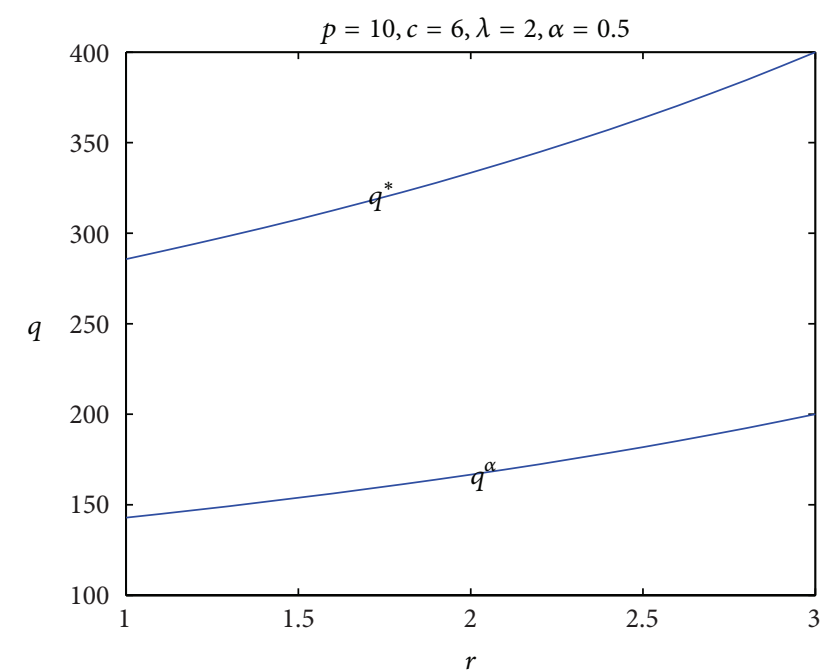

FIGURE 3: Optimal order quantities $q^{*}$ and $q^{\alpha}$ with different value of $r$.

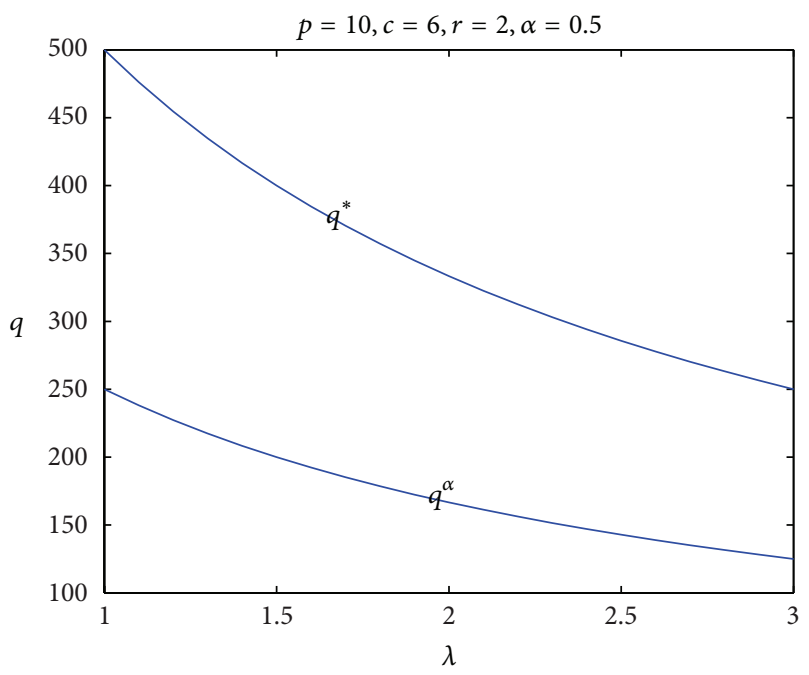

FIGURE 4: Optimal order quantities $q^{*}$ and $q^{\alpha}$ with different value of $\lambda$.

$p=10, c=6$, and $r=2$. For these parameters, let us compute the optimal order quantities $q^{*}$ and $q^{\alpha}$ obtained in Section 3 for the loss-averse newsvendor and give a sensitivity analysis.

First, let $\lambda=2$ and $\alpha=0.5$, we compute the optimal order quantities $q^{*}$ and $q^{\alpha}$ with different value of the retial price $p$, the wholesale price $c$, and the salvage price $r$ for the loss-averse newsvendor separately, and the results are given in Figures 7, 8, and 9, respectively.

By Figures 7, 8, and 9, it is easily checked that both $q^{*}$ and $q^{\alpha}$ are increasing in the retail price $p$ and the salvage price $r$ and decreasing in the wholesale price $c$. Moreover, it satisfies $q^{*}>q^{\alpha}$ for different value of $p, c$, and $r$.

Further, let $p=10, c=6, r=2$, and $\alpha=0.5$, we compute the optimal order quantities $q^{*}$ and $q^{\alpha}$ with different value of the loss aversion coefficient $\lambda$ for the loss-averse newsvendor,

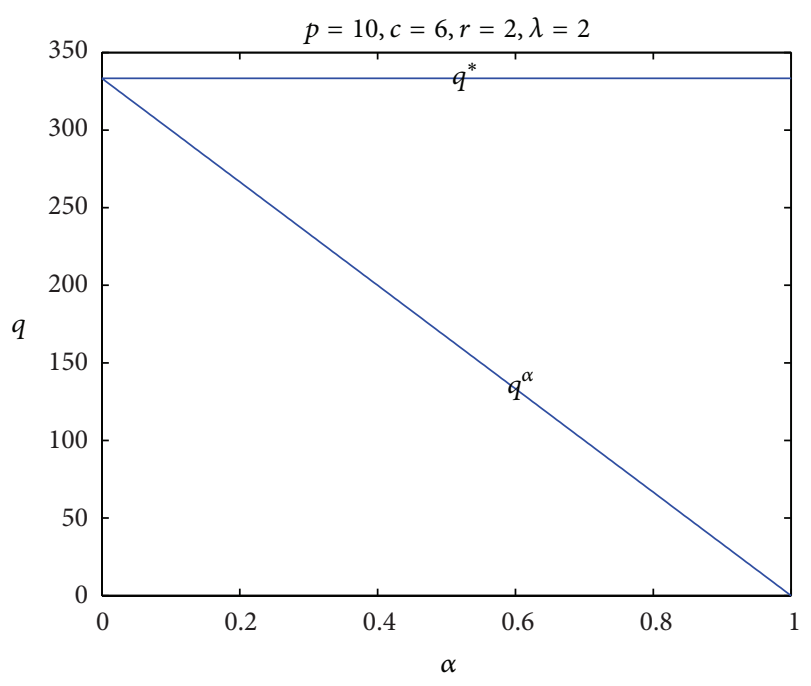

FIGURE 5: Optimal order quantities $q^{*}$ and $q^{\alpha}$ with different value of $\alpha$.

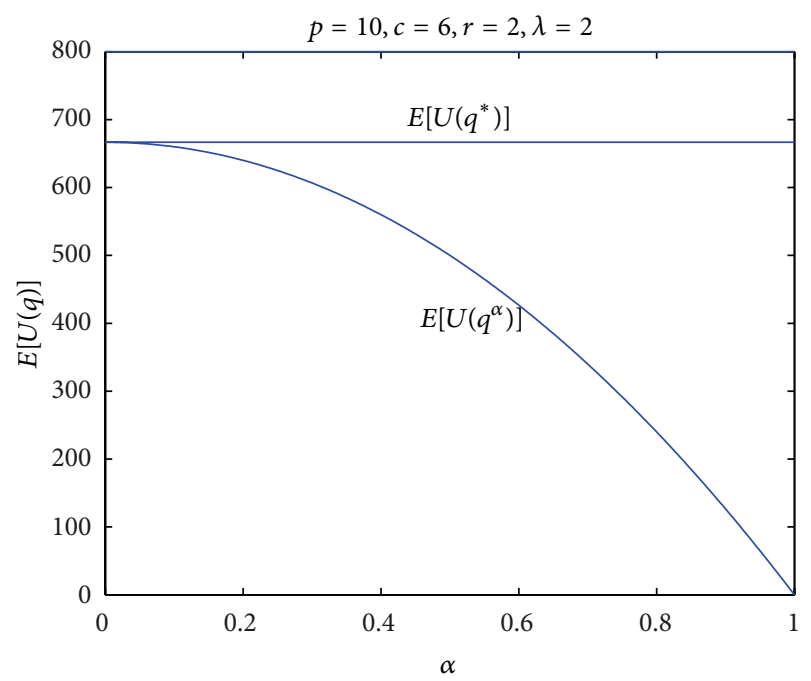

FIGURE 6: Expected utilities $E\left[U\left(q^{*}\right)\right]$ and $E\left[U\left(q^{\alpha}\right)\right]$ with different value of $\alpha$.

and the result is given in Figure 10. By Figure 10, the optimal order quantities $q^{*}$ and $q^{\alpha}$ both are decreasing in the loss aversion coefficient $\lambda$. Besides, it also satisfies $q^{*}>q^{\alpha}$ for different value of $\lambda$.

Then, let $p=10, c=6, r=2$, and $\lambda=2$, we compute the optimal order quantities $q^{*}$ and $q^{\alpha}$ with different value of the confidence level $\alpha$ for the loss-averse newsvendor, and the result is given in Figure 11. By Figure 11, the optimal order quantity $q^{*}(\alpha=0)$ stays the same and the optimal order quantity $q^{\alpha}$ is decreasing in the confidence level $\alpha$. Besides, it also satisfies $q^{*}>q^{\alpha}$ for different value of $\alpha$.

To summarize this section, the numerical results and sensitivity analysis confirm that the results obtained in Section 3 are qualitatively robust. There are following suggestions for the loss-averse newsvendor to choose an optimal order quantity to maximize his expected utility or CVaR about utility: 


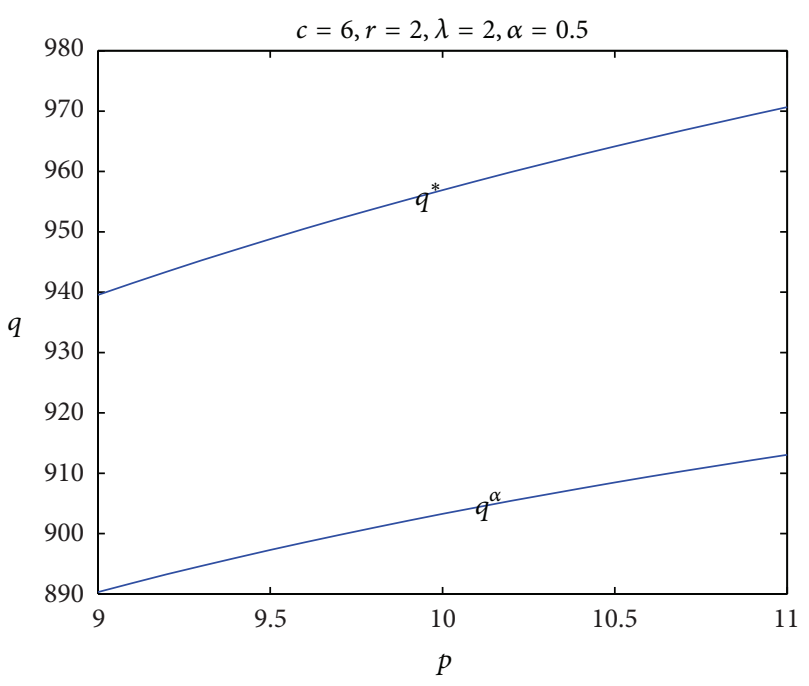

FIGURE 7: Optimal order quantities $q^{*}$ and $q^{\alpha}$ with different value of $p$.

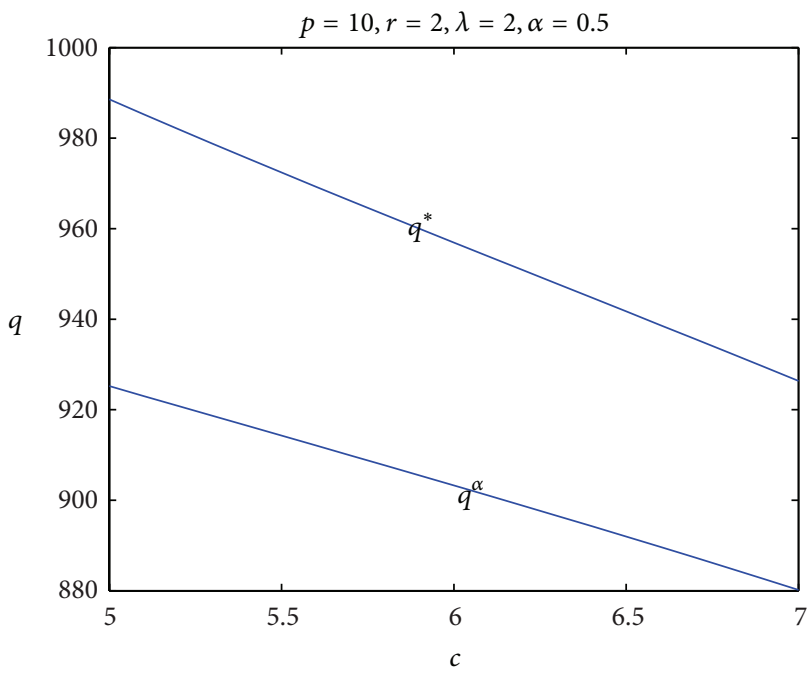

FigURE 8: Optimal order quantities $q^{*}$ and $q^{\alpha}$ with different value of c.

compared with the optimal order quantity for a loss-averse newsvendor who is risk-neutral to maximize his/her expected utility, the loss-averse newsvendor who is risk-averse had better ordered less products to reduce the risk originating from the fluctuation in the market demand. However, a lower order quantity which reduces the risk originating from the fluctuation in the market demand brings a lower expected utility for the loss-averse newsvendor, while a higher order quantity brings a higher expected utility that may produce more risk for the loss-averse newsvendor.

\section{Conclusions}

In the newsvendor model, some newsvendors are more averse to the losses (comeing from the excess order or lost sales) when the selling season is due than they are attracted to the same sized gains obtained in the selling season, which

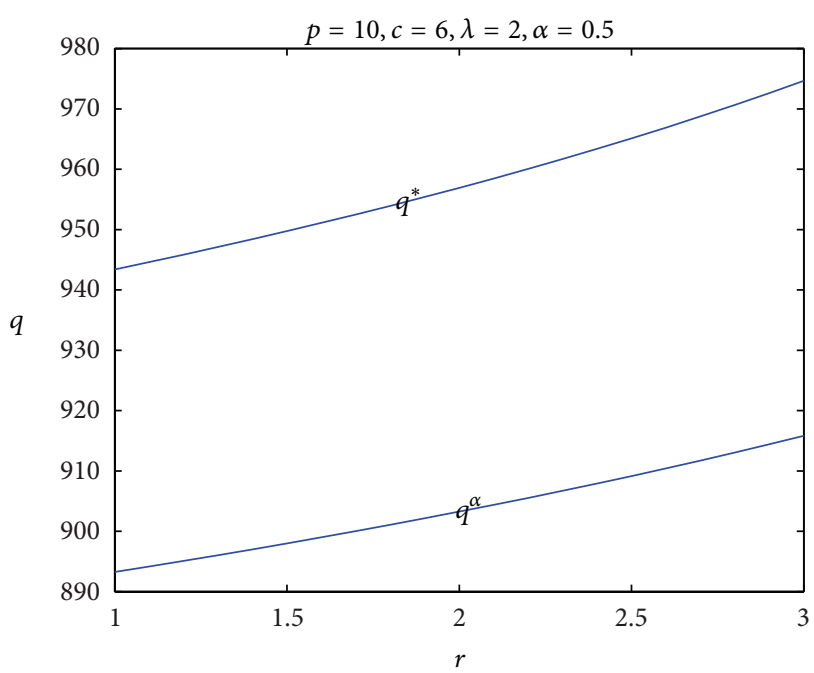

FIgURE 9: Optimal order quantities $q^{*}$ and $q^{\alpha}$ with different value of $r$.

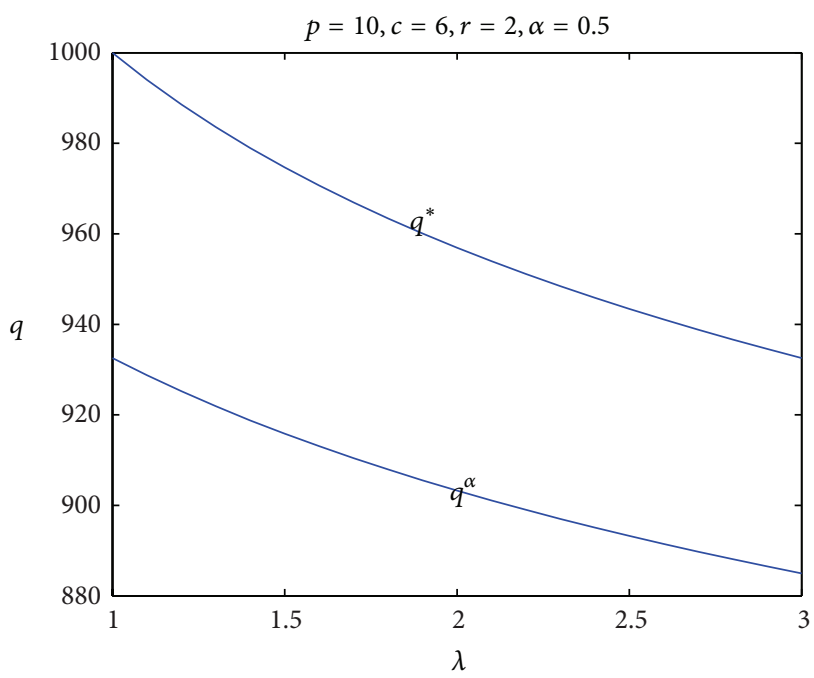

FIGURE 10: Optimal order quantities $q^{*}$ and $q^{\alpha}$ with different value of $\lambda$.

can be seen as the loss aversion in the newsvendor model. However, the study about the influence of loss aversion on the optimal order quantity decisions of the newsvendor model is very few. Then, this paper contributes to the study about the optimal order quantity decisions of such a lossaverse newsvendor model. By introducing the loss aversion coefficient $\lambda$, we introduce a novel utility function to address the loss aversion in the newsvendor model. Then, we achieve the optimal order quantities for the loss-averse newsvendor with different risk preferences to optimize different objectives about this utility function. We first obtain the optimal order quantity to maximize the expected utility for the loss-averse newsvendor who is risk-neutral and then obtain the optimal order quantity to maximize the CVaR about utility for the loss-averse newsvendor who is risk-averse, which can help the risk-averse newsvendor to reduce/control the risk originating from the fluctuation in the market demand. Our 


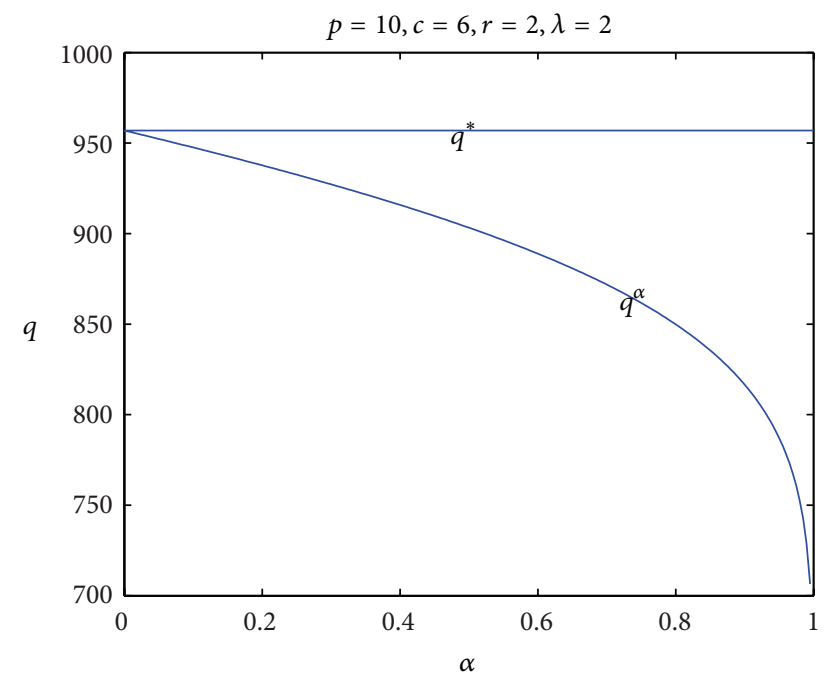

Figure 11: Optimal order quantities $q^{*}$ and $q^{\alpha}$ with different value of $\alpha$.

study find that the optimal order quantity for the loss-averse newsvendor to maximize his/her expected utility is smaller than the expected profit maximization order quantity in the classical newsvendor model, and this may help to explain the decision bias in the classical newsvendor model. Moreover, it is found that, in the loss-averse newsvendor model, if the loss-averse newsvendor is risk-averse, his/her optimal order quantity to maximize the CVaR about utility is decreasing in the confidence level $\alpha$, and the expected utility under such an optimal order quantity is decreasing in the confidence level $\alpha$ as well. This verifies that if the loss-averse newsvendor selects an order quantity to reduce/control the potential risk, he/she will expect a lower utility. Besides, it is shown that if the newsvendor becomes more loss-averse, then he/she will order less products to maximize his/her expected utility or CVaR about utility. Thus, this research shows how the loss aversion influence the optimal order quantity decisions in the newsvendor model and may present some policies to mitigate the decision bias in the classical newsvendor model.

Some extensions of this research are possible. For example, in this paper, the shortage penalty for the lost sales is not considered in defining the utility function for the lossaverse newsvendor. However, the loss from the lost sales that ranges from profit loss on the scale to some unspecific loss of goodwill of the customers has an important influence on the utility of the newsvendor, and then a possible extension is to integrate the shortage penalty for the lost sales into the definition of the utility function for the loss-averse newsvendor and then consider the optimal order quantities of the loss-averse newsvendor with different objectives about such a utility.

\section{Appendix}

Proof of Theorem 4. For a realized market demand $D$ and an order quantity $q$ of the newsvendor, by (9), we have

$$
U(q)=(p-c) q-[p-c+\lambda(c-r)](q-D)^{+} .
$$

Now, we define an auxiliary function

$$
\begin{aligned}
& h(q, v)=v-\frac{1}{1-\alpha} E[v-U(q)]^{+}=v-\frac{1}{1-\alpha} \\
& \cdot \int_{0}^{+\infty}[v-(p-c) q \\
& \left.\quad+(p-c+\lambda(c-r))(q-t)^{+}\right]^{+} d F(t)=v \\
& \quad-\frac{1}{1-\alpha} \int_{0}^{q}[v+\lambda(c-r) q \\
& \quad-(p-c+\lambda(c-r)) t]^{+} d F(t)-\frac{1}{1-\alpha} \int_{q}^{+\infty}[v \\
& \quad-(p-c) q]^{+} d F(t) .
\end{aligned}
$$

By the result in Rockafellar and Uryasev 2002, [32] (Section 3 , Corollary 11$), h(q, v)$ is jointly concave in $(q, v)$ since $P(q)$ is concave in $q$.

Then, by the result in Section 3, the optimal solution to problem $\left(\mathrm{P}_{1}\right)$ is equal to the optimal solution to the following problem:

$$
\max _{q \geq 0}\left[\max _{v \in R} h(q, v)\right] .
$$

Then, for any fixed $q$, we distinguish between the following cases.

Case $1(v \leq-\lambda(c-r) q)$. In this case, by (A.2), we have

$$
\begin{gathered}
h(q, v)=v, \\
\frac{\partial h(q, v)}{\partial v}=1>0 .
\end{gathered}
$$

Case $2(-\lambda(c-r) q \leq v \leq(p-c) q)$. In this case, by (A.2), we have

$$
\begin{aligned}
& h(q, v)=v-\frac{1}{1-\alpha} \int_{0}^{(v+\lambda(c-r) q) /(p-c+\lambda(c-r))}[v \\
& \quad+\lambda(c-r) q-(p-c+\lambda(c-r)) t] d F(t), \\
& \frac{\partial h(q, v)}{\partial v}=1-\frac{1}{1-\alpha} F\left[\frac{v+\lambda(c-r) q}{p-c+\lambda(c-r)}\right] .
\end{aligned}
$$

Obviously, it satisfies

$$
\left.\frac{\partial h(q, v)}{\partial v}\right|_{v=-\lambda(c-r) q}=1>0 .
$$


Then, if it satisfies

$$
\left.\frac{\partial h(q, v)}{\partial v}\right|_{v=(p-c) q}=1-\frac{1}{1-\alpha} F(q) \leq 0
$$

that is, $q \geq F^{-1}(1-\alpha)$; then by (A.6), the optimal solution $v^{*}$ to problem $\max _{v \in R} h(q, v)$ solves

$$
1-\frac{1}{1-\alpha} F\left[\frac{v^{*}+\lambda(c-r) q}{p-c+\lambda(c-r)}\right]=0
$$

which implies

$$
v^{*}=(p-c+\lambda(c-r)) F^{-1}(1-\alpha)-\lambda(c-r) q .
$$

Case $3(v \geq(p-c) q)$. In this case, by (A.2), we have

$$
\begin{aligned}
& h(q, v)=v-\frac{1}{1-\alpha} \int_{0}^{q}[v+\lambda(c-r) q \\
& \quad-(p-c+\lambda(c-r)) t] d F(t)-\frac{1}{1-\alpha} \int_{q}^{+\infty}[v \\
& \quad-(p-c) q] d F(t), \\
& \frac{\partial h(q, v)}{\partial v}=1-\frac{1}{1-\alpha} \leq 0 .
\end{aligned}
$$

Then, the optimal solution $v^{*}$ to problem $\max _{v \in R} h(q, v)$ is given as

$$
v^{*}=(p-c) q .
$$

Based on the analysis above, it is clear that, for any fixed $q$, the optimal solution $v^{*}$ to problem $\max _{v \in R} h(q, v)$ is given by

$$
v^{*}= \begin{cases}(p-c+\lambda(c-r)) F^{-1}(1-\alpha)-\lambda(c-r) q, & q \geq F^{-1}(1-\alpha), \\ (p-c) q, & q \leq F^{-1}(1-\alpha) .\end{cases}
$$

Then, to solve problem $\max _{q \geq 0}\left[\max _{v \in R} h(q, v)\right]=$ $\max _{q \geq 0} h\left(q, v^{*}\right)$, we distinguish between two different cases.

(i) Consider $q \geq F^{-1}(1-\alpha)$.

In this case, it follows from (A.13) that

$v^{*}=(p-c+\lambda(c-r)) F^{-1}(1-\alpha)-\lambda(c-r) q$.

Then by (A.2), we have

$$
\begin{aligned}
& h\left(q, v^{*}\right)=(p-c+\lambda(c-r)) F^{-1}(1-\alpha)-\lambda(c \\
& -r) q-\frac{1}{1-\alpha} \int_{0}^{F^{-1}(\alpha)}[(p-c+\lambda(c-r)) \\
& \left.\cdot\left(F^{-1}(\alpha)-t\right)\right] d F(t), \\
& \frac{\partial h\left(q, v^{*}\right)}{\partial q}=-\lambda(c-r)<0 .
\end{aligned}
$$

(ii) Consider $q \leq F^{-1}(1-\alpha)$.

In this case, it follows from (A.13) that $v^{*}=(p-c) q$.

Then by (2), we have

$$
\begin{aligned}
h\left(q, v^{*}\right) & \\
= & (p-c) q \\
& \quad-\frac{1}{1-\alpha} \int_{0}^{q}[(p-c+(c-r))(q-t)] d F(t),
\end{aligned}
$$

$$
\begin{aligned}
& \frac{\partial h\left(q, v^{*}\right)}{\partial q} \\
& \quad=p-c-\frac{1}{1-\alpha}[p-c+\lambda(c-r)] F(q) .
\end{aligned}
$$

Then it follows from (A.17) that the optimal solution $q^{\alpha}$ to problem $\max _{q \geq 0} h\left(q, v^{*}\right)$ is given as

$$
q^{\alpha}=F^{-1}\left[\frac{(1-\alpha)(p-c)}{p-c+\lambda(c-r)}\right] .
$$

This completes the proof.

\section{Conflict of Interests}

The authors declare that there is no conflict of interests regarding the publication of this paper.

\section{Acknowledgments}

The authors would like to thank the editor and the anonymous referee for their valuable suggestions and comments, which help us to improve this paper greatly. This research is supported by the Natural Science Foundation of Shandong Province with Grant ZR2014GQ005.

\section{References}

[1] C. X. Wang, "Random yield and uncertain demand in decentralised supply chains under the traditional and VMI arrangements," International Journal of Production Research, vol. 47, no. 7, pp. 1955-1968, 2009. 
[2] X. Li, Y. Li, and X. Cai, "Quantity decisions in a supply chain with early returns remanufacturing," International Journal of Production Research, vol. 50, no. 8, pp. 2161-2173, 2012.

[3] M. Ogier, V.-D. Cung, J. Boissière, and S. H. Chung, "Decentralised planning coordination with quantity discount contract in a divergent supply chain," International Journal of Production Research, vol. 51, no. 9, pp. 2776-2789, 2013.

[4] M. E. Schweitzer and G. P. Cachon, "Decision bias in the newsvendor problem with a known demand distribution: experimental evidence," Management Science, vol. 46, no. 3, pp. 404-420, 2000.

[5] A. O. Brown and C. S. Tang, "The impact of alternative performance measures on single-period inventory policy," Journal of Industrial and Management Optimization, vol. 2, no. 3, pp. 297318, 2006.

[6] L. Eeckhoudt, C. Gollier, and H. Schlesinger, "The risk averse (and prudent) newsboy," Management Science, vol. 41, no. 5, pp. 786-794, 1995.

[7] V. Agrawal and S. Seshadri, "Impact of uncertainty and risk aversion on price and order quantity in the newsvendor problem," Manufacturing and Service Operations Management, vol. 2, no. 4, pp. 410-423, 2000.

[8] X. Chen, M. Sim, D. Simichi-Levi, and P. Sun, Risk Aversion in Inventory Management, MIT Press, Cambridge, Mass, USA, 2003.

[9] J.-Y. Gotoh and Y. Takano, "Newsvendor solutions via conditional value-at-risk minimization," European Journal of Operational Research, vol. 179, no. 1, pp. 80-96, 2007.

[10] M. Xu and J. Li, "Optimal decisions when balancing expected profit and conditional value-at-risk in newsvendor models," Journal of Systems Science and Complexity, vol. 23, no. 6, pp. 1054-1070, 2010.

[11] S. Choi, A. Ruszczyński, and Y. Zhao, "A multiproduct riskaverse newsvendor with law-invariant coherent measures of risk," Operations Research, vol. 59, no. 2, pp. 346-364, 2011.

[12] A. H. Lau and H. Lau, "Maximizing the probability of achieving a target profit level in a two-product newsboy problem," Decision Sciences, vol. 19, no. 2, pp. 392-408, 1988.

[13] E. Sankarasubramanian and S. Kumaraswamy, "Optimal order quantity for pre-determined level of profit," Management Science, vol. 29, pp. 512-514, 1983.

[14] A. J. A. Bostian, C. A. Holt, and A. M. Smith, "Newsvendor 'pullto-center' effect: adaptive learning in a laboratory experiment," Manufacturing and Service Operations Management, vol. 10, no. 4, pp. 590-608, 2008.

[15] T. Feng, L. R. Keller, and X. Zheng, "Decision making in the newsvendor problem: a cross-national laboratory study," Omega, vol. 39, no. 1, pp. 41-50, 2011.

[16] N. Lau, S. Hasija, and J. N. Bearden, "Newsvendor pull-to-center reconsidered," Decision Support Systems, vol. 58, no. 1, pp. 6873, 2014.

[17] M. Khouja, "The single-period (news-vendor) problem: literature review and suggestions for future research," Omega, vol. 27, no. 5, pp. 537-553, 1999.

[18] Y. Qin, R. Wang, A. J. Vakharia, Y. Chen, and M. M. Seref, "The newsvendor problem: review and directions for future research," European Journal of Operational Research, vol. 213, no. 2, pp. 361-374, 2011.

[19] Z. Shapira, "Risk in managerial decision making," Working Paper, Hebrew University, 1986.
[20] K. R. MacCrimmon and D. A. Wehrung, Taking Risks: The Management of Uncertainty, Free Press, New York, NY, USA, 1996.

[21] S. Benartzi and R. H. Thaler, "Myopic loss aversion and the equity premium puzzle," Quarterly Journal of Economics, vol. 110, pp. 73-92, 1995.

[22] D. S. Putler, "Incorporating reference price effects into a theory of household choice," Marketing Science, vol. 11, no. 3, pp. 287309, 1992.

[23] A. Fiegenbaum and H. Thomas, "Attitudes toward risk and the risk-return paradox: prospect theory explanations," Academy of Management Journal, vol. 31, no. 1, pp. 85-106, 1988.

[24] C. Camerer, L. Babcock, G. Loewenstein, and R. H. Thaler, "Labor supply of New York city cabdrivers: one day at a time," Quarterly Journal of Economics, vol. 112, no. 2, pp. 406-441, 1997.

[25] E. Soltani, A. Azadegan, Y.-Y. Liao, and P. Phillips, "Quality performance in a global supply chain: finding out the weak link," International Journal of Production Research, vol. 49, no. 1, pp. 269-293, 2011.

[26] W. Liu, S. J. Song, and C. Wu, "Impact of loss aversion on the newsvendor game with product substitution," International Journal of Production Economics, vol. 141, no. 1, pp. 352-359, 2013.

[27] F. Herweg, "The expectation-based loss-averse newsvendor," Economics Letters, vol. 120, no. 3, pp. 429-432, 2013.

[28] N. Neumann and U. Böckenholt, "A meta-analysis of loss aversion in product choice," Journal of Retailing, vol. 90, no. 2, pp. 182-197, 2014.

[29] P. Artzner, F. Delbaen, J. M. Eber, and D. Heath, "Coherent measures of risk," Mathematical Finance, vol. 9, no. 3, pp. 203228, 1999.

[30] H. Mauser and D. Rosen, "Beyond VaR: from measuring risk to managing risk," ALGO Research Quarterly, vol. 1, pp. 5-20, 1999.

[31] R. T. Rockafellar and U. Stanislav, "Optimization of conditional value-at-risk,” Journal of Risk, vol. 2, pp. 21-41, 2000.

[32] R. T. Rockafellar and S. Uryasev, "Conditional value-at-risk for general loss distributions," Journal of Banking \& Finance, vol. 26, no. 7, pp. 1443-1471, 2002. 


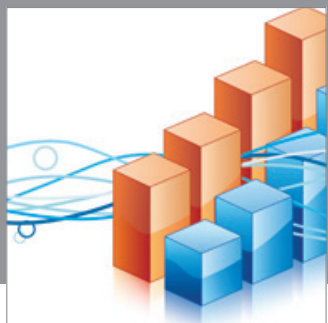

Advances in

Operations Research

mansans

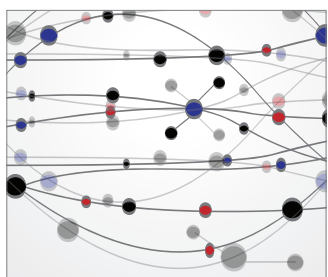

The Scientific World Journal
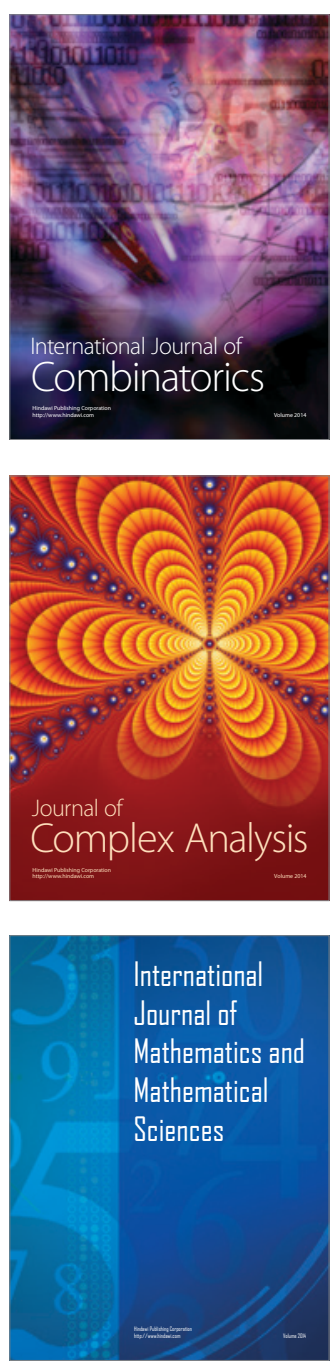
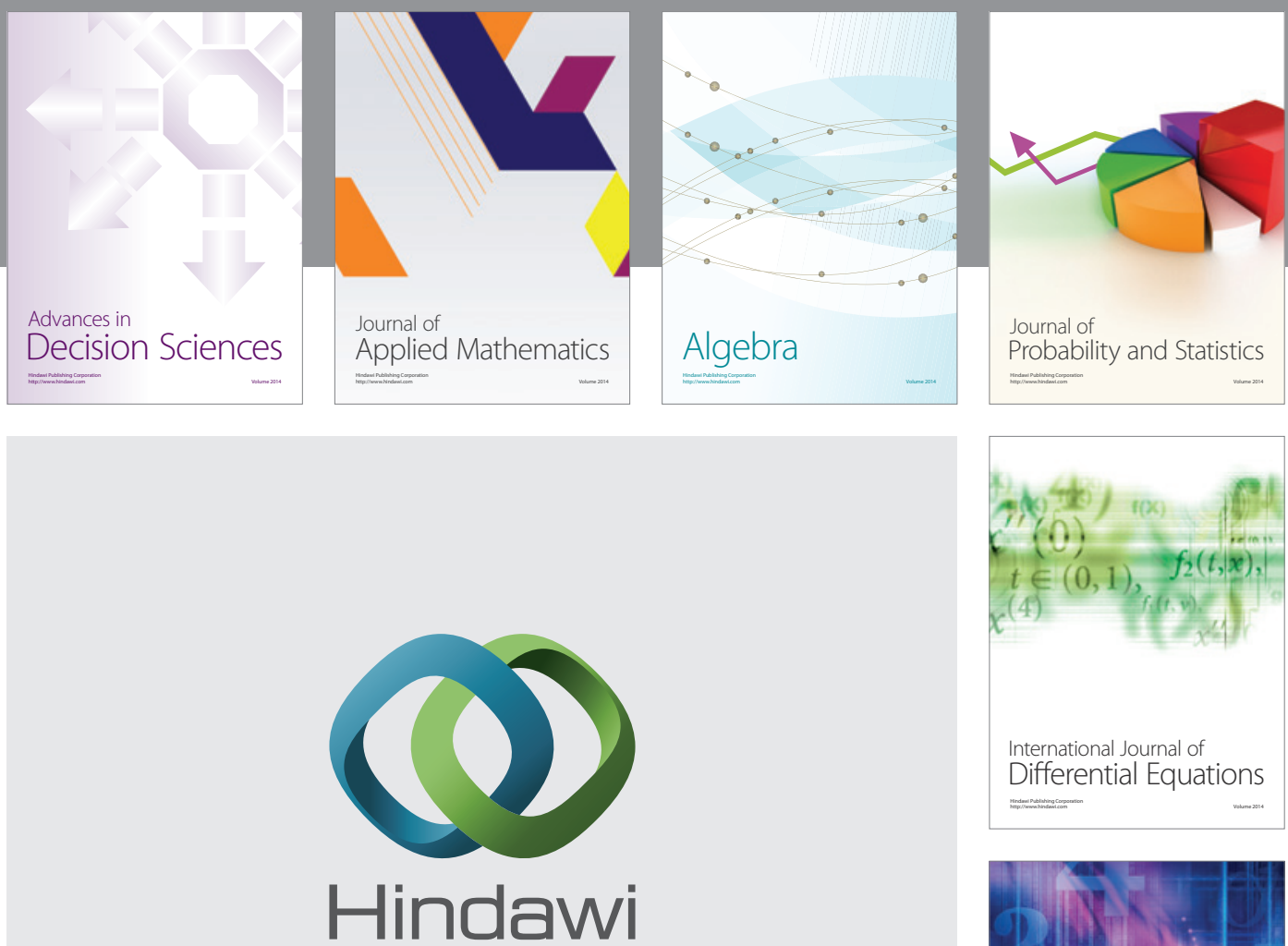

Submit your manuscripts at http://www.hindawi.com
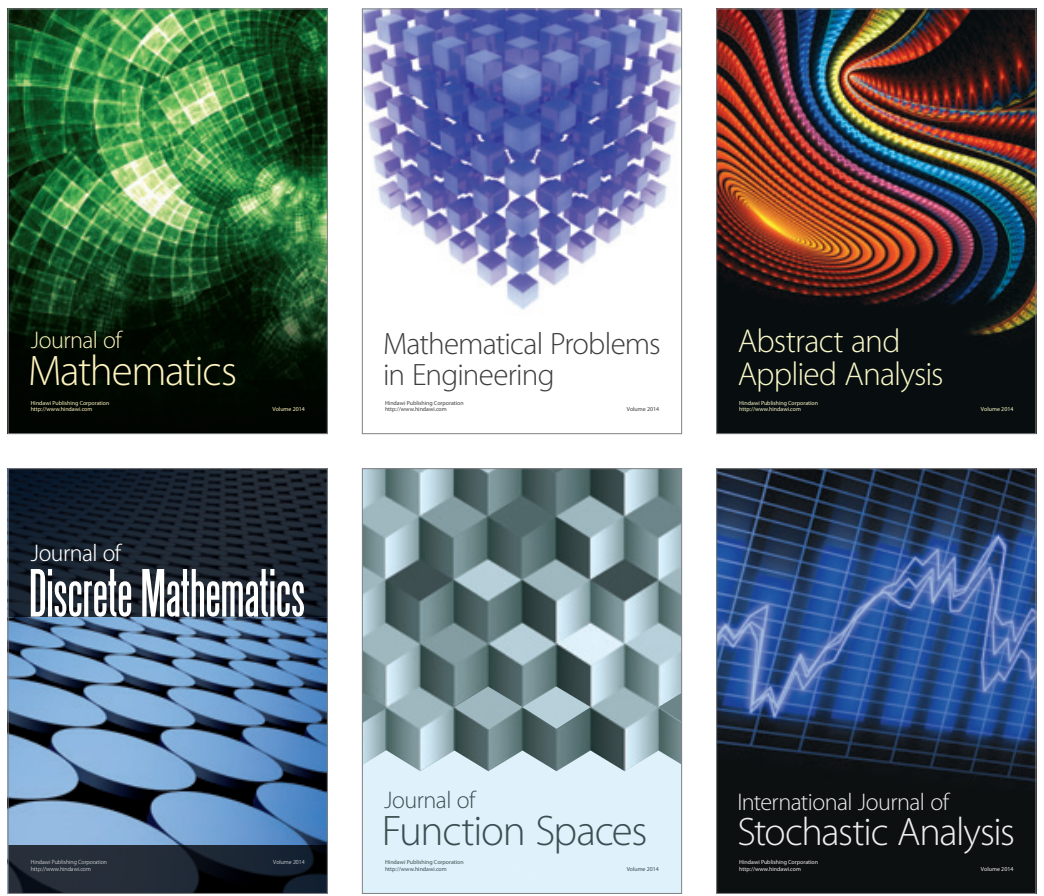

Journal of

Function Spaces

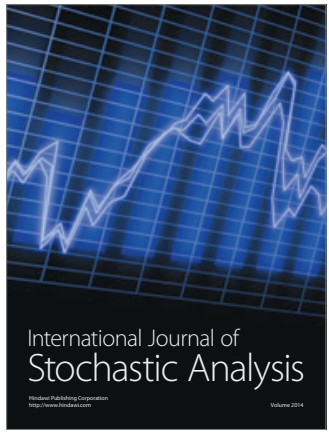

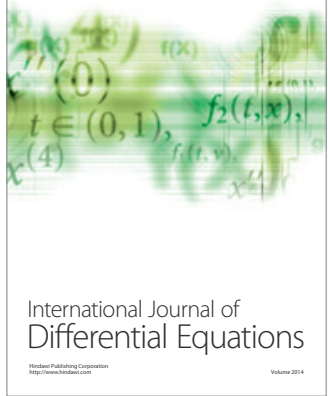
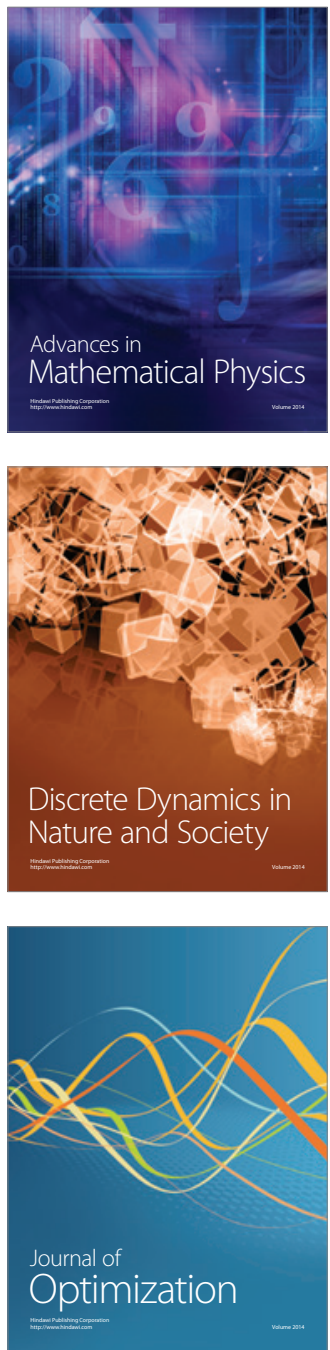